\title{
Cancer-Related Self-Disclosure in the Workplace/School by Adolescent and Young Adult Cancer Survivors
}

\author{
Carolyn Rabin, $\mathrm{PhD}$
}

Purpose: Little is known about how survivors of adolescent and young adult (AYA) cancers navigate the process of communicating their cancer history to those at work/school. The purpose of this study was to evaluate the factors impacting workplace/school disclosure decisions among AYA survivors and strategies used for self-disclosure. Two hypotheses were tested: (1) survivors would be significantly less likely to disclose their cancer history and more likely to avoid cancer-related topics at work/school versus with friends/acquaintances; (2) survivors' satisfaction with previous cancer-related self-disclosures at work/school would mediate the relationship between the reactions of others to those disclosures and the likelihood of future work/school disclosures.

Methods: Online survey data, including a measure of self-disclosure adapted from the Nebraska Outness Scale, were collected from 120 survivors (age $=15$ to 39 years at diagnosis, 19 to 45 years when surveyed). Data were analyzed descriptively and study hypotheses were evaluated using paired $t$-tests (Hypothesis 1) and multiple regression with bootstrapping (Hypothesis 2).

Results: Both hypotheses were supported: participants were less likely to self-disclose at work/school than to friends/acquaintances $(p<0.001)$ and satisfaction with previous self-disclosure at work/school mediated the relationship between reactions to previous disclosures and likelihood of future disclosures $(95 \%$ confidence interval $=0.39-0.81$ ). Descriptive analyses indicated that participants were guided by a variety of factors when making disclosure decisions (e.g., anticipated reactions) and used a range of strategies for disclosures (e.g., humor).

Conclusions: AYA survivors are cautious in their approach to workplace/school disclosure. Employers and school administrators should create supportive and inclusive environments that facilitate self-disclosure for survivors who wish to self-disclose.

Keywords: workplace, self-disclosure, employment, cancer history

\section{Introduction}

A DOLESCENT AND YOUNG ADULT (AYA) cancer survivors who pursue employment or education after a cancer diagnosis must decide if, when and how to disclose their cancer status in the workplace or at school. Although not all AYA survivors pursue employment or education, one study found that $72 \%$ of those employed or in school before diagnosis returned to work/school. ${ }^{1}$ These survivors must learn to navigate the process of self-disclosure in a way that will provide them access to any needed support or accommodations but will not hinder their advancement during the formative years of their career. Given the relative rarity of a cancer history among young adults, AYA survivors are unlikely to have peer role models who can guide them through cancer-related professional disclosures. ${ }^{2,3}$ Thus, this study focused on developing an understanding of how AYA survivors navigate these disclosures to determine whether any additional support or guidance is needed.

Research on cancer-related disclosure in the workplace suggests that disclosure decisions differ across survivors and contexts. When 192 cancer survivors were asked whether they would disclose their cancer history when applying for a new position, $43 \%$ indicated that they would in some situations but not others. ${ }^{4}$ In another study, men who had been diagnosed with prostate cancer expressed reluctance to disclose their diagnosis to others but also an obligation to tell supervisors to explain absences from work. ${ }^{5}$ Survivors in a qualitative study echoed the need to make disclosures when work-related absences were anticipated; these survivors noted, however, that they would be less likely to disclose at work if no health-related accommodations were needed. ${ }^{6}$

Clinical Psychology Department, William James College, Newton, Massachusetts, USA. 
Research also indicates that survivors are more likely to selfdisclose at work if they tend to disclose this information in other contexts, perceive their workplace environment to be supportive, or hope to garner support from coworkers. 6,7

Survivors who choose to communicate with others at work/school about their cancer experience may reap physical and psychosocial benefits. Self-disclosure has been associated with enhanced mood and life satisfaction among survivors (although this cross-sectional research precludes conclusions about causality) and enhanced health and immune functioning in the general population. ${ }^{8-10}$ Furthermore, one study found that adult survivors of childhood cancer who disclosed their cancer status at work were less likely to report an intention to leave their job. ${ }^{7}$

AYA survivors may be wary of making self-disclosures in the workplace/school, however, given the potential for stigmatization and discrimination. Research on self-disclosure in the general population indicates that disclosing something that others see as a weakness may tarnish perceptions of the discloser. ${ }^{11}$ This may explain research demonstrating that employers underestimate the abilities of employees with a cancer history and overestimate the degree to which they will be impacted by fatigue, emotional distress, and physical symptoms. ${ }^{12}$ Likewise, qualitative research indicates that while employers do not believe that cancer survivors are stigmatized in the workplace, survivors feel highly stigmatized and believe their abilities and productivity are underestimated. ${ }^{6}$ Unfortunately, there is also evidence that the stigmatization experienced by cancer survivors may result in workplace discrimination. An experimental study found that when job applicants identified as cancer survivors, they were significantly less likely to be called in response to their application and more likely to experience "passive harm" from managers (e.g., avoidance of eye contact, rudeness, and hostility). ${ }^{4}$ Likewise, in qualitative research, survivors described workplace discrimination including bullying, refusing accommodations, and constrained opportunities for career advancement. ${ }^{6}$

Thus, although disclosing one's cancer history is associated with positive outcomes, making such disclosures in the workplace/school may be risky. A variety of individual and system-level factors-including reliance of the survivor's family on his/her income, inadequate disability protections, and temporary employment status-may make disclosure unwise for some survivors. As little is currently known about how AYA survivors navigate the disclosure process, this study aimed to assess the factors shaping workplace/school self-disclosure decisions, strategies used for self-disclosure, and the type of information disclosed. Two hypotheses were tested. The first was that AYA survivors would be less likely to disclose their cancer status and more likely to avoid topics related to their cancer when interacting with people at work/school versus friends/acquaintances. The second hypothesis was that AYA survivors who experienced a more positive reaction to previous disclosures at work/school would feel more satisfied with those disclosures and, consequently, be more likely to anticipate making future workplace/school self-disclosures. The latter hypothesis was based on disclosure decision-making models and research suggesting that individuals are more likely to disclose "secrets" when they anticipate a positive reaction and/or have received a positive reaction in the past. ${ }^{13-16}$ In addition, previous research with AYA survivors indicates that their satisfaction with the self-disclosure experience mediates the relationship between the reaction of peers to self-disclosure and the likelihood of disclosing to peers again in the future. ${ }^{17}$ Thus, similar relationships were hypothesized for workplace/school self-disclosure. (Note that, although disclosure decisions may differ across workplace and school contexts, these were assessed collectively given the wording of the available self-disclosure measure. ${ }^{18}$ )

\section{Methods}

Institutional review board approval was obtained before initiating this research (IRB no. 20170030). Participants were recruited with the help of an organization serving AYA survivors; this organization posted a study advertisement and link to the online survey on social media. Survivors who clicked on the link were asked to confirm that they had been diagnosed with cancer (excluding nonmelanoma skin cancer) and were between 18 and 45 years of age. After confirming that they met inclusion criteria, survivors were directed to an online consent form and brief survey. Participants were offered a $\$ 20$ incentive for participating.

\section{Measures}

The survey included a measure assessing the following demographic and cancer-related information: age, gender identity, marital status, race, ethnicity, education, employment (whether currently employed, homemaker, student, etc.), household income, cancer diagnosis, age at diagnosis, and cancer treatments. Participants were also asked to complete the measures described hereunder.

\section{Level of cancer self-disclosure}

As validated measures of cancer self-disclosure are not currently available, a validated scale measuring sexual orientation disclosure was adapted. The Nebraska Outness Scale $^{18}$ (NOS) contains a five-item self-disclosure subscale asking participants to estimate the percentage of people in five different groups (e.g., people at work/school, friends/ acquaintances) aware of their sexual orientation. The wording of this subscale was modified for this study to read, "What percent of the people in each group below do you think are aware that you were diagnosed with cancer?" Participants indicated the percentage-from $0 \%$ to $100 \%$ for each group of people. The NOS also contains a five-item concealment subscale asking participants to rate the degree to which they avoid talking about topics related to sexual orientation with members of the same five groups of people. The wording of the concealment subscale was modified for this study to read, "How often do you avoid talking about topics related to your cancer (e.g., your health, cancer diagnosis, the effects of your cancer treatment) when you are with members of these groups?' Participants rated levels of concealment with each group of people on a scale from "Never (0)" to "Always (10)." The reliability of the original scale was 0.89 and internal consistency ranged from 0.87 to $0.92^{18}$; the internal consistency of the adapted scale was 0.76 . Hypothesis 1 was evaluated using ratings of disclosure to and concealment from those at work/school and friends/acquaintances. 


\section{Reasons for self-disclosing or not disclosing}

Participants were asked to indicate the reasons they had sometimes disclosed their cancer history to different groups of people including people at work/school (e.g., "It was important for them to know about me"). Participants were then asked to identify the reasons they had sometimes not disclosed to people in each group (e.g., "So they wouldn't see or treat me differently"). For a list of the response choices for both items, see Table 2 . In the absence of available measures to assess constructs of interest for this study, this measure and those described below were based on survivorship and decision-making literature ${ }^{6,7,13-15}$ and researcher expertise.

\section{Type of information disclosed}

Participants were asked to identify which of the following cancer-related information they had disclosed to different groups of people, including people at work/school: diagnosis, type of treatment, treatment effects, and the emotional aspects of having cancer.

\section{Strategies used for self-disclosure}

Participants were asked to identify which strategies they used when disclosing their cancer status to different groups of people (e.g., "Reassured them that I am okay"); for a complete list of response choices, see Table 2.

\section{Postdisclosure experiences}

Participants rated how different groups of people had reacted to their self-disclosures using 0-to-10 scales from "very badly-e.g., got upset, did not seem interested (0)" to "very well-e.g., listened, were supportive (10)." Participants then rated how satisfied they were with the experience of selfdisclosing to each group using 0-to-10 scales from "not at all satisfied (0)" to "very satisfied (10)." Finally, participants rated how likely they were to self-disclose to each group of people in the future using 0-to-10 scales from "not at all likely (0)" to "very likely (10)." Participants were also given the opportunity to indicate if any of these items were not applicable. Hypothesis 2 was evaluated using ratings of others' reactions to work/school disclosures, satisfaction with previous work/school disclosures, and likelihood of future disclosure at work/school.

\section{Data analysis}

SPSS version 24.0.0.0 was used for data analysis. Descriptive analyses were performed to evaluate the factors guiding workplace/school self-disclosure decisions among AYA survivors, type of information disclosed, and strategies used when self-disclosing. Hypothesis 1 was then evaluated with two paired $t$-tests. One $t$-test compared the percentage of people at work/school that survivors had disclosed their cancer status to with the percentage of friends/acquaintances. The second $t$-test compared survivors' ratings of concealment among people at work/school versus friends/acquaintances. Hypothesis 2 was evaluated using multiple regression with bootstrapping. ${ }^{19,20}$ This approach was used to determine whether ratings of how those at work/school had reacted to past self-disclosure (i.e., the predictor variable) were related to participants' reported likelihood of future workplace/ school self-disclosure (i.e., the outcome variable) through the hypothesized mediator of satisfaction with previous workplace/school self-disclosure. Bootstrapping, computed for each of 5000 samples using a 95\% confidence interval (CI), was used to estimate whether this indirect/mediated effect was significant.

\section{Results}

Analyses were conducted on data from 120 participants. Although 199 launched the survey, only 137 provided data and 15 of these ceased participation after responding to a limited number of items. The 15 "drop outs" did not differ significantly from the remaining 122 with respect to demographic characteristics [i.e., age $(p=0.27)$, gender $(p=0.98)$, marital status $(p=0.66)$, race $(p=0.47)$, ethnicity $(p=0.39)$, income $(p=0.27)$, employment $(p=0.96)$, and education $(p=0.09$; effect size $=0.28)]$. Two additional participants were removed from the data before analysis as they did not

Table 1. Demographic Characteristics OF THE SAMPLE $(n=120)$

\begin{tabular}{|c|c|c|}
\hline & $\mathrm{n}$ & $\%$ \\
\hline \multicolumn{3}{|l|}{ Gender } \\
\hline Female & 96 & 80.0 \\
\hline Male & 24 & 20.0 \\
\hline \multicolumn{3}{|l|}{ Race $^{\mathrm{a}}$} \\
\hline White & 107 & 89.2 \\
\hline More than one race & 7 & 5.8 \\
\hline Asian & 4 & 3.3 \\
\hline \multicolumn{3}{|l|}{ Ethnicity } \\
\hline Non-Hispanic & 102 & 85.0 \\
\hline Hispanic & 18 & 15.0 \\
\hline \multicolumn{3}{|l|}{ Education } \\
\hline Bachelor's degree & 44 & 36.7 \\
\hline Graduate school degree & 38 & 31.7 \\
\hline Some college & 16 & 13.3 \\
\hline Associates degree & 15 & 12.5 \\
\hline High school diploma & 4 & 3.3 \\
\hline Vocational or trade school & 3 & 2.5 \\
\hline \multicolumn{3}{|l|}{ Marital status } \\
\hline Married & 50 & 41.7 \\
\hline Single & 44 & 36.7 \\
\hline Living with partner & 17 & 14.2 \\
\hline Divorced & 8 & 6.7 \\
\hline Separated & 1 & 0.8 \\
\hline \multicolumn{3}{|l|}{ Employment status } \\
\hline Employed full-time & 61 & 50.8 \\
\hline Unemployed & 18 & 15.0 \\
\hline Employed part-time & 16 & 13.3 \\
\hline Homemaker & 10 & 8.3 \\
\hline Student & 10 & 8.3 \\
\hline Medical leave & 4 & 3.3 \\
\hline Retired & 1 & 0.8 \\
\hline \multicolumn{3}{|l|}{ Annual household income ${ }^{a}$} \\
\hline$\$ 70,000$ and above & 54 & 45.0 \\
\hline$\$ 50,000-\$ 69,999$ & 25 & 20.8 \\
\hline$\$ 30,000-\$ 49,999$ & 18 & 15.0 \\
\hline$\$ 10,000-\$ 29,999$ & 14 & 11.7 \\
\hline$<\$ 10,000$ & 8 & 6.7 \\
\hline
\end{tabular}

${ }^{a}$ Two participants did not respond to the item on race and one participant to the item on income. 
meet the National Cancer Institute definition of an AYA survivor (i.e., older than 39 years at diagnosis).

The demographic characteristics of the remaining 120 participants are detailed in Table 1. Participants ranged in age from 15 to 39 years at diagnosis (mean, $M=27.1$, standard deviation, $\mathrm{SD}=6.0)$ and 19 to 45 when surveyed $(\mathrm{M}=31.7$, $\mathrm{SD}=6.1)$. Most identified as White $(89.2 \%)$, non-Hispanic $(85.0 \%)$, and women $(80.0 \%)$. Most were employed fulltime or part-time $(64.2 \%)$ with a small percentage in school $(8.3 \%)$. Although the remainder reported being unemployed, homemakers, on medical leave or retired, some of these participants may have completed items on workplace/school self-disclosure based on previous work/school experiences. Participants were diagnosed with a range of cancers, an average of 4.8 years before study participation. Breast cancer was the most prevalent diagnosis.

Table 2 details the reasons participants sometimes choose to disclose or not disclose their cancer status to people at work/school and strategies used for self-disclosure. The most commonly reported reasons for self-disclosure were that survivors were discussing a related topic or felt it was important for others to know about them. The most commonly

Table 2. Percentage of Participants Endorsing

EACH Reason for Disclosing or Not Disclosing to People at Work/School, Using Each Disclosure Strategy and Disclosing Each Type OF INFORMATION

n $\%$

Reason to disclose

We were talking about a related topic (e.g., my 5243.3 health)

It was important for them to know about me $51 \quad 42.5$

I thought they would respond well and be $\quad 4739.2$ supportive

I needed their help with something cancerrelated (e.g., ride to doctor, time off work)

N/A-I have not told [people at work/school] about my cancer

Reasons to not disclose

It was none of their business

So they wouldn't see or treat me differently

To avoid upsetting or burdening them

So they wouldn't distance themselves

They wouldn't have responded well or been supportive

N/A-I told [all my friends] about my cancer

Disclosure strategies

Only provided the information they asked for

Reassured them that I am ok (or will be ok)

Used humor to keep things light

Did not tell them some of the scarier information

Waited until a time I thought they could handle 3529.2 it

Information disclosed

Cancer diagnosis

Cancer treatment

$95 \quad 79.2$

$83 \quad 69.2$

Treatment effects (including long-term such as $48 \quad 40.0$ physical disability, infertility, fatigue)

Emotional aspects of cancer experience

$22 \quad 18.3$

N/A, not applicable. reported reason for not self-disclosing at work/school were that survivors felt that it was not others' business or so that they would not be seen or treated differently. The most frequently used strategies for disclosure were: only providing information that was specifically requested, reassuring others that the survivor was/would be okay and humor. When survivors made workplace/school disclosures, they were most likely to disclose information about their cancer diagnosis, treatment, and treatment effects; survivors least often discussed the emotional aspects of their cancer experience.

Hypothesis 1 proposed that AYA survivors would be less likely to self-disclose their cancer status and more likely to avoid topics related to their cancer when interacting with people at work/school versus friends/acquaintances. Survivors reported that, on average, they had self-disclosed to $57.6 \%$ of those they knew at work/school but $70.5 \%$ of their friends/acquaintances. A paired $t$-test confirmed that participants had self-disclosed to a significantly smaller percentage of people at work/school $(\mathrm{M}=57.6, \quad \mathrm{SD}=30.9)$ than friends/acquaintances $(\mathrm{M}=70.5, \mathrm{SD}=22.9) ; t(115)=-5.84$, $p<0.001$. A second paired $t$-test indicated that participants were significantly more likely to avoid topics related to their cancer when speaking with people at work/school $(\mathrm{M}=52.5$, $\mathrm{SD}=32.1)$ versus friends/acquaintances $(\mathrm{M}=44.8, \mathrm{SD}=30.1)$; $t(117)=4.43, p<0.001$. Thus, Hypothesis 1 was supported.

Regression and bootstrapping was used to evaluate Hypothesis 2-that is, that AYA survivors who received more positive reactions to previous cancer-related disclosures at work/school would feel more satisfied with those disclosures and, consequently, be more likely to anticipate making future disclosures at work/school. As given in Figure 1, standardized regression coefficients indicated a significant relationship between the reactions of those at work/school to previous disclosures and satisfaction with workplace/school self-disclosure $(0.78, p<0.001)$ and between satisfaction with workplace/school self-disclosure and likelihood of future workplace/school disclosure $(0.78, p<0.001)$. The bootstrapped indirect effect of the reactions of those at work/school on the likelihood of future workplace/school disclosures as mediated by survivors' satisfaction with workplace/school self-disclosure was also statistically significant (0.61) using a 95\% CI (0.39-0.81). Thus, Hypothesis 2 was also supported.

\section{Discussion}

The AYA survivors in this study demonstrated a fair amount of restraint in their workplace/school selfdisclosures. Survivors often disclosed only information that was specifically requested and limited their disclosures to factual information (e.g., cancer diagnosis or treatment rather than cancer-related emotions). These findings are consistent with previous research indicating that survivors are careful not to disclose more than necessary. ${ }^{5,6}$ Likewise, findings indicate that AYA survivors were less comfortable making cancer-related disclosures at work/school than when among friends, possibly because of fears of workplace/school discrimination. $^{4,6,12}$ Indeed, half the participants indicated that they sometimes choose not to disclose their cancer status at work/school to avoid being seen or treated differently (It is noteworthy, however, that participants only self-disclosed to a mean of $70.5 \%$ of friends/acquaintances; thus, $100 \%$ workplace/school self-disclosure should not be expected.). 


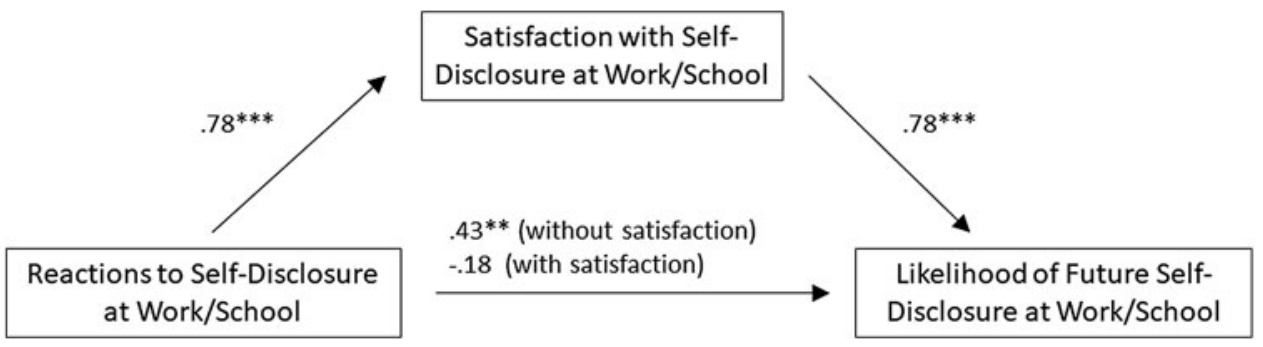

FIG. 1. Mediational model in which satisfaction with self-disclosure to people at work/school mediates the relationship between reactions of people at work/school to self-disclosure and survivors' estimated likelihood of future disclosure at work/school.

Although discriminatory treatment may be a concern for any cancer survivor, the risk may be particularly acute for young adults who are less established in their careers and more dependent on the benevolence of supervisors and mentors to advance.

Although concern about discrimination may have limited workplace/school disclosures, disclosure decisions were also shaped by other factors. These included the perceived relevance of the information to the conversation or relationship, the anticipated reactions from others, and past disclosure experiences. Those who received positive reactions to previous workplace/school disclosures were more likely to feel satisfied with the experience and to anticipate making similar disclosures in the future. These findings parallel previous research on the factors guiding AYA survivors' self-disclosure to friends. ${ }^{14}$ Thus, AYA survivors appear to make self-disclosure decisions based, not only on anticipated reactions from others (as suggested by disclosure decision-making models), but also the reactions received to past disclosures. ${ }^{14,15}$

Findings from this study should be interpreted within the context of study limitations. The sample was fairly homogenous-predominantly women and White. (With respect to ethnicity, however, nearly $15 \%$ identified as Hispanic, which is close to the percentage in the U.S. population.) A methodological weakness is that the adapted version of the NOS, used to measure cancer-related self-disclosure, has not been validated. In addition, this measure asked participants to report on their disclosure experiences in aggregate. This may have impacted findings as participants might have made different disclosure decisions over time (e.g., during vs. after treatment) and in different work/school environments. It is also unclear what participants who were not currently employed or in school were referencing when responding to workplace/school self-disclosure items (although they may have responded based on previous experiences). In addition, given that a minority of participants were currently in school and type of employment (e.g., contract work, long-term employment) was not assessed, it is unclear whether findings adequately represent the experience of disclosure in school or different work contexts. Likewise, findings may not generalize internationally to countries in which there is a more protective welfare system.

The findings from this study suggest some potentially fruitful avenues for future research. The adapted version of the NOS used in this study might be further modified to capture additional information (e.g., assess workplace and school self-disclosure separately). In addition, future studies might evaluate specific discrimination-related concerns (e.g., fear of dismissal) that may make AYA survivors reluctant to self-disclose at work. Future research should also explore the role of demographic factors in shaping disclosure decisions.
For example, exploratory analyses not reported here suggest that ethnicity may play a role in disclosure-with Hispanic participants indicating greater openness to workplace/school self-disclosure - although re-running this study's mediational analysis with ethnicity did not change results.

Thus, AYA survivors appear to make disclosure decisions based on a variety of factors including individual experience and contextual variables. Although some may appropriately conclude that it is unwise to self-disclose (e.g., as they are in unsupportive or discriminatory work/school environments), others may want guidance to navigate a necessary or desired disclosure. Both AYA survivors and their employers/schools may benefit from interventions to educate them about survivors' rights to reasonable accommodations and protection from discrimination under the Americans with Disabilities Act. Creating more inclusive, supportive environments for AYA survivors will enable each to make an individual choice about self-disclosure in the absence of fear of the response.

\section{Author Disclosure Statement}

No competing financial interests exist.

\section{Data Availability Statement}

The data that support the findings of this study are available from the corresponding author upon reasonable request.

\section{Funding Information}

This project was supported by a Faculty Seed Grant from William James College.

\section{References}

1. Parsons HM, Harlan LC, Lynch CF, et al. Impact of cancer on work and education among adolescent and young adult cancer survivors. J Clin Oncol. 2012;30:2393-400.

2. Reuben SH. Living beyond cancer: finding a new balance. Bethesda, MD: U.S. Department of Health and Human Services, National Institutes of Health, National Cancer Institute; 2004. Accessed March 24, 2020 from: http:// deainfo.nci.nih.gov/advisory/pcp/pcp03-04rpt/survivorship .pdf.

3. Gloeckler Ries LA, Reichman ME, Lewis DR, et al. Cancer survival and incidence from the Surveillance, Epidemiology and End Results (SEER) program. Oncologist. 2003; 8(6):541-52.

4. Martinez LR, White CD, Shapiro JR, Hebl MR. Selection BIAS: stereotypes and discrimination related to having a history of cancer. J Appl Psychol. 2016;101(1):122-8.

5. Gray RE, Fitch M, Phillips C, et al. To tell or not to tell: patterns of disclosure among men with prostate cancer. Psychooncology. 2000;9:273-82. 
6. Stergiou-Kita M, Pritlove C, Kirsh B. The "Big C"sigma, cancer, and workplace discrimination. J Cancer Surviv. 2016;10:1035-50.

7. Martinez LR, Hebl MR. Adult survivors of childhood cancers' identity disclosures in the workplace. J Cancer Surviv. 2016;10:416-24.

8. Park CL, Bharadwaj AK, Blank TO. Illness centrality, disclosure, and well-being in younger and middle-aged adult cancer survivors. Br J Health Psychol. 2011;16:880-9.

9. Pennebaker JW, O'Heeron RC. Confiding in others and illness rate among spouses of suicide and accidental-death victims. J Abnorm Psychol. 1984;93(4):473-6.

10. Pennebaker JW, Kiecolt-Glaser JK, Glaser R. Disclosure of traumas and immune function: health implications for psychotherapy. J Consult Clin Psychol. 1988;56(2): 239-45.

11. Gibson KR, Harari D, Marr JC. When sharing hurts: how and why self-disclosing weakness undermines the taskoriented relationships of higher status disclosers. Organ Behav Hum Decis Process. 2017;144:25-43.

12. Grunfeld EA, Low E, Cooper AF. Cancer survivors' and employers' perceptions of working following cancer treatment. Occup Med. 2010;60:611-7.

13. Chaudoir SR, Fisher JD. The disclosure processes model: understanding disclosure decision-making and post-disclosure outcomes among people living with a concealable stigmatized identity. Psychol Bull. 2011;136(2):236-56.

14. Greene K, Magsamen-Conrad K, Ventis MK, et al. Assessing health diagnosis disclosure decisions in relation- ships: testing the disclosure decision-making model. Health Commun. 2012;27:356-68.

15. Magsamen-Conrad K. Dimensions of anticipated reaction in information management: anticipating responses and outcomes. Rev Commun. 2014;14:314-33.

16. Kelly AE, McKillop KJ. Consequences of revealing personal secrets. Psychol Bull. 1996;120(3):450-65.

17. Rabin C. Self-disclosure to peers by young adult cancer survivors. Psychooncology. 2018;28:181-6.

18. Meidlinger PC, Hope DA. Differentiating disclosure and concealment in measure of outness for sexual minorities: the Nebraska Outness Scale. Psychol Sex Orientat Gend Divers. 2014;1(4):489-97.

19. Hayes AF. Beyond Baron and Kenny: statistical mediation analysis in the new millennium. Commun Monogr. 2009; 76(4):408-20.

20. Hayes AF. Introduction to mediation, moderation, and conditional process analysis, 2nd edition. New York, NY: Guilford Press; 2017.

Address correspondence to: Carolyn Rabin, PhD Clinical Psychology Department William James College 1 Wells Avenue Newton, MA 02459 $U S A$

Email: carolyn_rabin@williamjames.edu 